\title{
Planar cell polarity pathway and development of the human visual cortex
}

Jean Shin ${ }^{1,2}$, Shaojie $\mathrm{Ma}^{3}$, Edith Hofer ${ }^{4,5}$, Yash Patel ${ }^{2}$, Gennady V. Roshchupkin ${ }^{6,7,8}$, André M. Sousa ${ }^{9}$, Xueqiu Jian ${ }^{10}$, Rebecca Gottesman ${ }^{11}$, Thomas H. Mosley ${ }^{12}$, Myriam Fornage $^{10}$, Yasaman Saba ${ }^{13}$, Lukas Pirpamer ${ }^{4}$, Reinhold Schmidt ${ }^{4}$, Helena Schmidt ${ }^{13}$, Amaia Carrion-Castillo ${ }^{14}$, Fabrice Crivello ${ }^{15}$, Bernard Mazoyer ${ }^{15}$, Joshua C. Bis ${ }^{16}$, Shuo $\mathrm{Li}^{17}$, Qiong Yang ${ }^{17}$, Michelle Luciano ${ }^{18,19}$, Sherif Karama ${ }^{20}$, Lindsay Lewis ${ }^{20}$, Mark Bastin $^{18,21}$, Mathew A. Harris ${ }^{21,22}$, Joanna M. Wardlaw ${ }^{18,23}$, lan E. Deary ${ }^{18,19}$, Markus Scholz ${ }^{24,25}$, Markus Loeffler ${ }^{24,25}$, Veronica Witte ${ }^{26,27}$, Frauke Beyer ${ }^{26,27}$, Arno Villringer ${ }^{26,28}$, Nicola J Armstrong ${ }^{29}$, Karen A. Mather ${ }^{30,31}$, David Ames $^{32,33}$, Jiyang Jiang ${ }^{30}$, John B Kwok $^{34,35}$, Peter R. Schofield ${ }^{35,36}$, Anbupalam Thalamuthu ${ }^{30}$, Julian N. Trollor ${ }^{30,37}$, Margaret J. Wright ${ }^{38,39}$, Henry Brodaty ${ }^{30,40}$, Wei Wen ${ }^{30}$, Perminder S. Sachdev ${ }^{30,41}$ ', Natalie Terzikhan ${ }^{8}$, Tavia E. Evans ${ }^{6,8}$, Hieab H.H.H. Adams ${ }^{6,8}$, M. Arfan Ikram ${ }^{6,8,42}$, Stefan Frenzel ${ }^{43}$, Sandra van der Auwera-Palitschka ${ }^{43,44}$, Katharina Wittfeld ${ }^{43,44}$, Robin Bülow $^{45}$, Hans Jörgen Grabe ${ }^{43,44}$, Christophe Tzourio ${ }^{46,47}$, Aniket Mishra ${ }^{46}$, Sophie Maingault $^{48}$, Stephanie Debette ${ }^{46,47,49}$, Nathan A. Gillespie ${ }^{50}$, Carol E. Franz ${ }^{51,52}$, William S. Kremen ${ }^{51,52,53}$, Linda Ding ${ }^{54}$, Neda Jahanshad ${ }^{54}$, the ENIGMA Consortium, Nenad Sestan $^{3}$, Zdenka Pausova $^{1}$, Sudha Seshadri ${ }^{49,55}$, Tomas Paus ${ }^{2,56}$; for the neuroCHARGE Working Group

${ }^{1}$ The Hospital for Sick Children, University of Toronto, Toronto, ON, Canada;

${ }^{2}$ Holland Bloorview Kids Rehabilitation Hospital, Bloorview Research Institute, University of Toronto, Toronto, ON, Canada;

${ }^{3}$ Departments of Genetics and Neuroscience, Yale University School of Medicine, New Haven, CT, USA;

${ }^{4}$ Clinical Division of Neurogeriatrics, Department of Neurology, Medical University of Graz, Austria;

${ }^{5}$ Institute for Medical Informatics, Statistics and Documentation, Medical University of Graz, Austria;

${ }^{6}$ Department of Radiology and Nuclear Medicine, Erasmus University Medical Center, Rotterdam, the Netherlands;

${ }^{7}$ Department of Medical Informatics, Erasmus MC, Rotterdam, The Netherlands

${ }^{8}$ Department of Epidemiology, Erasmus University Medical Center, Rotterdam, the Netherlands;

${ }^{9}$ Department of Neuroscience and Kavli Institute for Neuroscience, Yale School of Medicine, New Haven, CT, USA;

${ }^{10}$ Institute of Molecular Medicine and Human Genetics Center, University of Texas Health Science Center at Houston, Houston, TX, USA

${ }^{11}$ Johns Hopkins School of Medicine, Baltimore, MD, USA;

${ }^{12}$ University of Mississippi Medical Center, MS, USA;

${ }^{13}$ Gottfried Schatz Research Center for Cell Signaling, Metabolism and Aging, Medical University of Graz, Graz, Austria;

${ }^{14}$ Language and Genetics Department, Max Planck Institute for Psycholinguistics, Nijmegen, The Netherlands;

${ }^{15}$ Groupe d'Imagerie Neurofonctionnelle, Institut des Maladies Neurodégénératives, Centre National de la Recherche Scientifique, Commissariat à l'Energie Atomique, et Université de Bordeaux, Bordeaux, France;

${ }^{16}$ Cardiovascular Health Research Unit, Department of Medicine, University of Washington, Seattle, WA, USA

${ }^{17}$ Department of Biostatistics, Boston University School of Public Health, MA, USA; 
${ }^{18}$ Centre for Cognitive Epidemiology and Cognitive Ageing, University of Edinburgh, Edinburgh, UK;

${ }^{19}$ Department of Psychology, University of Edinburgh, Edinburgh, UK;

${ }^{20}$ Montreal Neurological Institute, McGill University, Montreal, QC, Canada;

${ }^{21}$ Centre for Clinical Brain Sciences, University of Edinburgh, Edinburgh, UK;

${ }^{22}$ Division of Psychiatry, University of Edinburgh, Edinburgh, UK;

${ }^{23}$ UK Dementia Research Institute, University of Edinburgh, Edinburgh, UK;

${ }^{24}$ Institute for Medical Informatics, Statistics and Epidemiology, University of Leipzig, Leipzig, Germany;

${ }^{25}$ LIFE Research Center for Civilization Diseases, Leipzig, Germany;

${ }^{26}$ Department of Neurology, Max Planck Institute of Cognitive and Brain Sciences, Leipzig, Germany;

${ }^{27}$ Faculty of Medicine, CRC 1052 Obesity Mechanisms, University of Leipzig, Leipzig, Germany;

${ }^{28}$ Day Clinic for Cognitive Neurology, University Hospital Leipzig, Leipzig, Germany;

${ }^{29}$ Mathematics and Statistics, Murdoch University, Perth, Australia;

${ }^{30}$ Centre for Healthy Brain Ageing, School of Psychiatry, University of New South Wales, Sydney, Australia;

${ }^{31}$ Neuroscience Research Australia, Sydney, Australia;

${ }^{32}$ National Ageing Research Institute, Royal Melbourne Hospital, VIC, Australia;

${ }^{33}$ Academic Unit for Psychiatry of Old Age, St. Vincent's Health, The University of Melbourne, VIC, Australia;

${ }^{34}$ Brain and Mind Centre - The University of Sydney, Camperdown, NSW, Australia;

${ }^{35}$ School of Medical Sciences, University of New South Wales, Sydney, Australia;

${ }^{36}$ Neuroscience Research Australia, Sydney Australia;

${ }^{37}$ Department of Developmental Disability Neuropsychiatry, School of Psychiatry, University of New South Wales, Sydney, Australia;

${ }^{38}$ Queensland Brain Institute, The University of Queensland, St Lucia, QLD, Australia;

${ }^{39}$ Centre for Advanced Imaging, The University of Queensland, St Lucia, QLD, Australia;

${ }^{40}$ Dementia Centre for Research Collaboration, University of New South Wales, Sydney, NSW, Australia;

${ }^{41}$ Neuropsychiatric Institute, Prince of Wales Hospital, Sydney, Australia;

${ }^{42}$ Department of Neurology, Erasmus MC University Medical Centre, Rotterdam, The Netherlands;

${ }^{43}$ Department of Psychiatry and Psychotherapy, University Medicine Greifswald, Germany;

${ }^{44}$ German Center for Neurodegenerative Diseases (DZNE), Site Rostock/ Greifswald, Germany;

${ }^{45}$ Institute for Diagnostic Radiology and Neuroradiology, University Medicine Greifswald, Greifswald, Germany;

${ }^{46}$ University of Bordeaux, Inserm, Bordeaux Population Health Research Center, team VINTAGE, UMR 1219, F-33000 Bordeaux, France;

${ }^{47} \mathrm{CHU}$ de Bordeaux, Department of Neurology, F-33000 Bordeaux, France;

${ }^{48}$ University of Bordeaux, Institut des Maladies Neurodégénratives, UMR5293, CEA, CNRS, Ubordeaux, F-33000 Bordeaux France;

${ }^{49}$ Department of Neurology, Boston University School of Medicine, Boston, MA, USA;

${ }^{50}$ Virginia Institute for Psychiatric and Behavioural Genetics, Virginia Commonwealth University, Richmond, VA, USA;

${ }^{51}$ Department of Psychiatry, University of California, San Diego, La Jolla, CA, USA;

${ }^{52}$ Center for Behavior Genetics of Aging, University of California, San Dieog, La Jolla, CA, USA; 
${ }^{53}$ VA San Diego Center of Excellence for Stress and Mental Health, La Jolla, California, USA;

${ }^{54}$ Imaging Genetics Center, Mark and Mary Stevens Neuroimaging and Informatics Institute, Keck School of Medicine of USC, University of Southern California, Los Angeles, CA, USA;

${ }^{55}$ Department of Epidemiology and Biostatistics, Glenn Biggs Institute for Alzheimer's and Neurodegenerative Diseases, UT Health San Antonio, San Antonio, USA;

${ }^{56}$ Departments of Psychology and Psychiatry, University of Toronto, Toronto, ON, Canada.

The radial unit hypothesis provides a framework for global (proliferation) and regional (distribution) expansion of the primate cerebral cortex ${ }^{1}$. Using principal component analysis (PCA), we have identified cortical regions with shared variance in their surface area and cortical thickness, respectively, segmented from magnetic resonance images obtained in 23,800 participants. We then carried out meta-analyses of genome-wide association studies of the first two principal components for each phenotype. For surface area (but not cortical thickness), we have detected strong associations between each of the components and single nucleotide polymorphisms in a number of gene loci. The first (global) component was associated mainly with loci on chromosome 17 (9.5e-32 $\leq p \leq$ $2.8 \mathrm{e}-10)$, including those detected previously as linked with intracranial volume ${ }^{2,3}$ and/or general cognitive function ${ }^{4}$. The second (regional) component captured shared variation in the surface area of the primary and adjacent secondary visual cortices and showed a robust association with polymorphisms in a locus on chromosome 14 containing Disheveled Associated Activator of Morphogenesis 1 (DAAM1; $p=2.4 \mathrm{e}-34)$. DAAM1 is a key component in the planar-cell-polarity signaling pathway, ${ }^{5,6}$. In follow-up studies, we have focused on the latter finding and established that: (1) DAAM1 is highly expressed between $12^{\text {th }}$ and $22^{\text {nd }}$ post-conception weeks in the human cerebral cortex; (2) genes co-expressed with DAAM1 in the primary visual cortex are enriched in mitochondriarelated pathways; and (3) volume of the lateral geniculate nucleus, which projects to regions of the visual cortex staining for cytochrome oxidase (a mitochondrial enzyme), correlates with the surface area of the visual cortex in major-allele homozygotes but not in carriers of the minor allele. Altogether, we speculate that, in concert with thalamocortical input to cortical subplate, DAAM1 enables migration of neurons to cytochrome-oxidase rich regions of the visual cortex, and, in turn, facilitates regional expansion of this set of cortical regions during development.

Using magnetic resonance imaging, one can derive a number of metrics informative with regard to development and aging of the human cerebral cortex, including cortical surface area and cortical thickness. The two measures provide insights into different developmental processes, each with a different timeline. Cortical surface area reflects primarily the tangential growth of the cerebral cortex during prenatal development; the phase of symmetric division of progenitor cells in the proliferative zones during the first trimester is particularly important for the tangential growth through additions of ontogenetic columns ${ }^{1}$. The subsequent phase of asymmetric division continues to increase the number of ontogenetic columns (and thus surface area) but it also begins to contribute to the thickness of cerebral cortex formed by post-mitotic neurons migrating from the proliferative zones to the cortical plate in the inside-out manner ${ }^{1}$. Ionizing radiation of the (monkey) fetus during early gestation reduces surface area (sparing cortical thickness) while the same radiation applied in midgestation affects both the surface area and cortical thickness ${ }^{7}$. While surface area remains stable after early 
childhood, cortical thickness continues to change, in particular during puberty and aging. Furthermore, both surface area and cortical thickness vary across individuals in global and regional manner.

Here we report findings obtained in 23,800 participants assessed across 19 cohorts from the CHARGE Consortium and the UK Biobank (Table E1 in Extended Data), and replicated in a subsequent release of the UK Biobank participants $(n=6,234)$ as well as in silico ${ }^{8}$ using region-based summary statistics provided by the ENIGMA Consortium $(n=19,512)$. To identify genetic loci associated with global and regional variations in each cortical phenotype, i.e., the surface area and thickness of the cerebral cortex, we have first carried out principal component analysis of regional values (34 regions segmented by FreeSurfer) in each cohort. For each phenotype, the first (PC1) and second (PC2) components loaded in similar sets of cortical regions across all 13 cohorts (Figures E1 [surface area] and E2 [cortical thickness] in Extended Data). Figure 1 illustrates the loadings for each of the 34 cortical regions in PC1 (Fig. 1A) and PC2 (Fig. 1B) for surface area. Note that PC2 includes only a handful of cortical regions in the medial aspect of the occipital lobe, including the pericalcarine (primary visual) cortex.

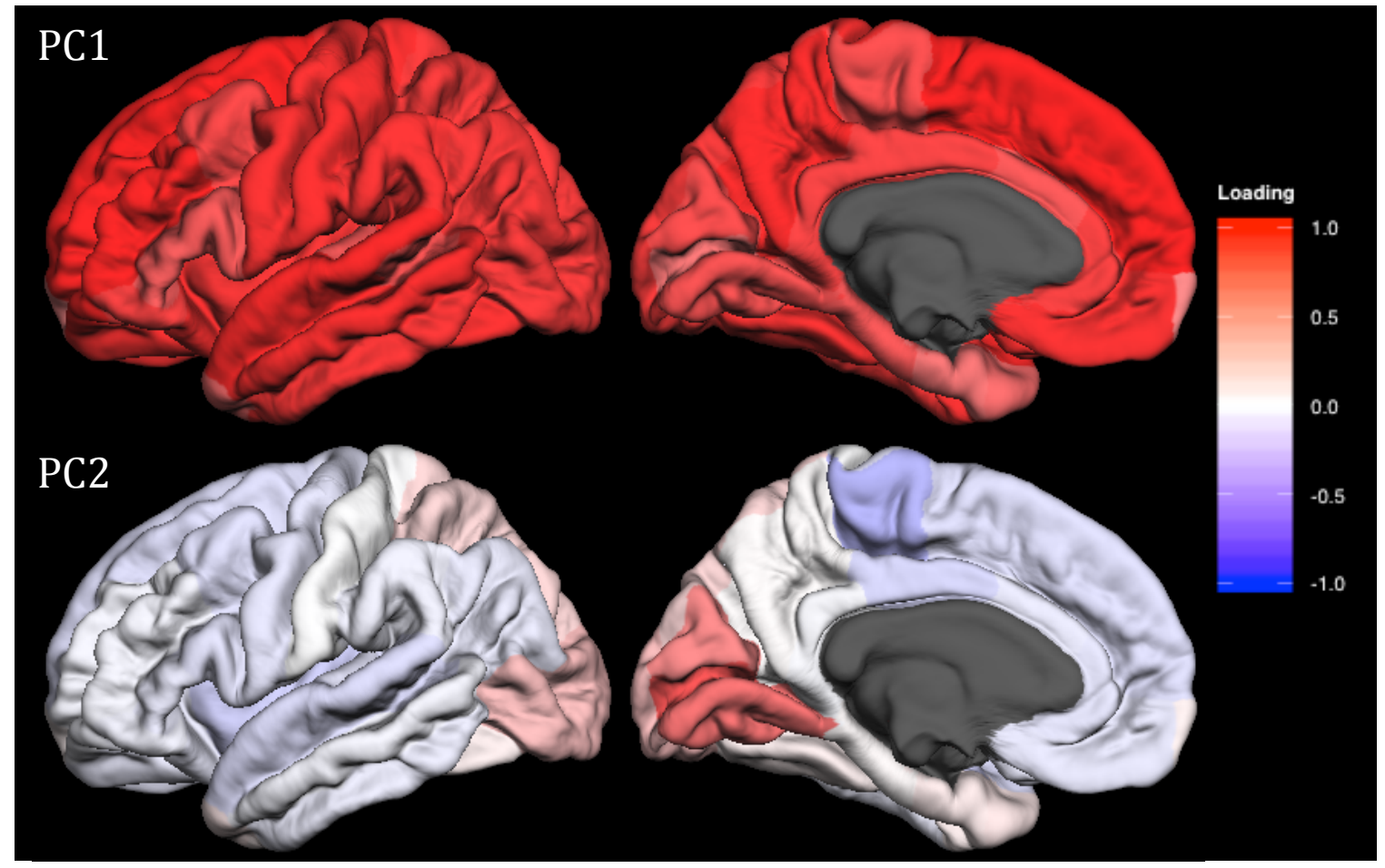

Figure 1. Lateral and medial views of median unrotated principal component (PC) loadings for the surface area of the $\mathbf{3 4}$ cortical regions in CHARGE consortium cohorts. Lateral (left column) and medial (right column) views of the median PC loadings are shown for PC1 (top) and PC2 (bottom). Each cohort estimated the surface area of the 34 cortical regions (left and right hemispheres summed), using FreeSurfer and carried out principal component analyses to obtain PC1 and PC2 loadings. Then, for each cortical region, median value of loadings was obtained across the cohorts. The red-to-blue color indicates the positive-to-negative loading values (i.e., correlation between PC scores and raw data) as indicated by the color bar. The median loading values were then used to derive the 'general' PC score for each individual and later used as the response variable in the GWAS meta-analyses.

We then executed a genome-wide association analysis (GWAS) in each of the 19 cohorts and, subsequently, meta-analyzed these cohort-based results (Supplementary 
Information) for each of the four phenotypes, namely PC1 and PC2 of surface area, and PC1 and PC2 of cortical thickness. For surface area, the first ("global") component was associated mainly with a number of loci on chromosome 17, including those detected previously as linked with intracranial volume ${ }^{2,3}$ [and/or general cognitive function ${ }^{4}$ ] (Figure E3A and Table E2A in Extended Data). The second ("regional") component was associated mainly with a locus on chromosome 14 containing DAAM1 (Figure 2, Figures $\mathrm{E} 3 \mathrm{~B}$ and E4, and Table E2B in Extended Data). For cortical thickness, meta-GWAS revealed a single locus associated with $\mathrm{PC} 1$ and a single locus associated with $\mathrm{PC} 2$ (Figure E5). The DAAM1 locus (top hit: rs73313052) was associated with surface area (but not cortical thickness) of each of the four cortical regions loading on PC2 when examined on a region-by-region basis, as reported in two independent reports from the CHARGE and ENIGMA Consortia (Table E3 in Extended Data).

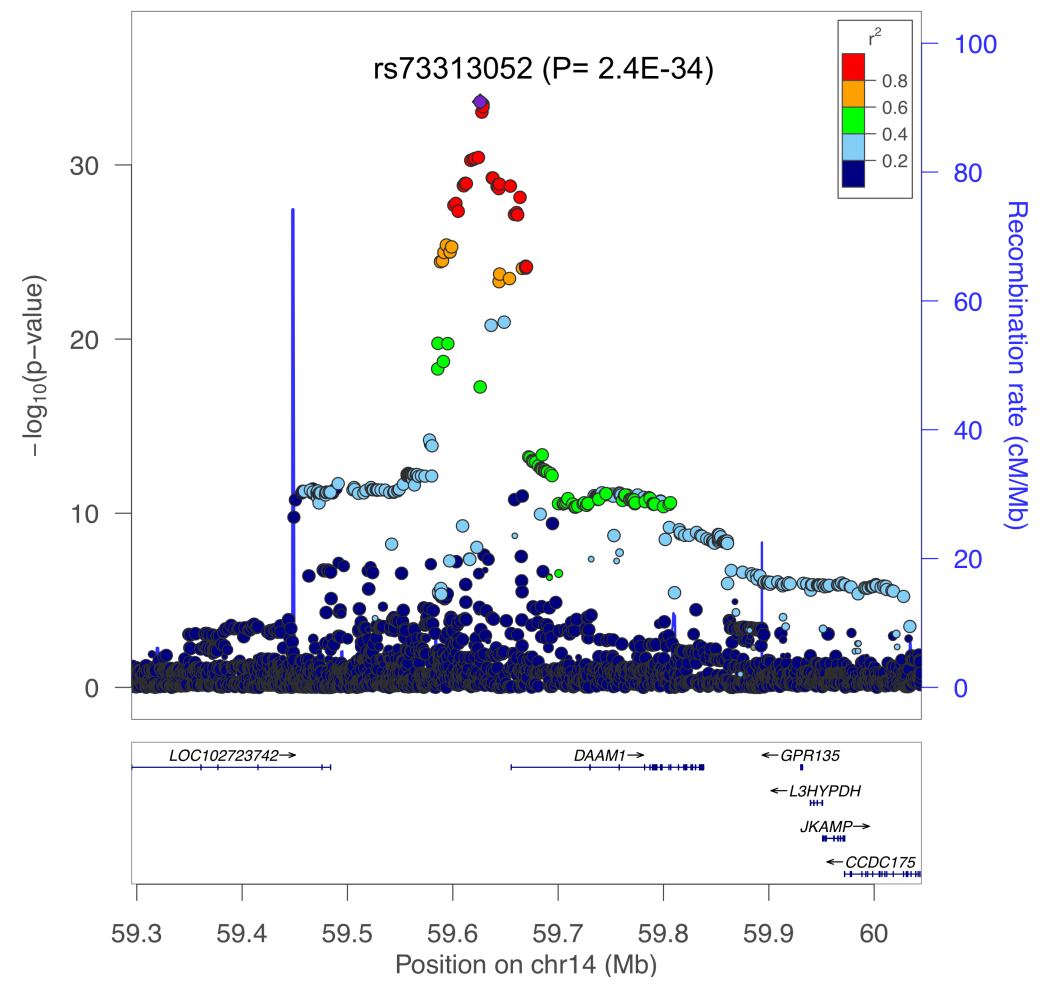

Figure2. Regional association plot for rs73313052, the top PC2 single nucleotide polymorphism (SNP). Each point indicates a SNP tested in the meta genome-wide association study (GWAS) of surface area PC2 within the shown genomic region. The top PC2 SNP is indicated by the purple diamond. The horizontal axis represents the genomic position on the human chromosome 14 (hg19). The left-vertical axis indicates the $-\log _{10} P$ values obtained from the GWAS meta-analysis (on the left side); and the right-vertical axis, the estimated recombination rate from the HapMap samples. The red-to-blue colors indicate the degree of linkage disequilibrium (LD) between each SNP and rs73313052. The LD was based on the pairwise squared allelic correlation $r^{2}$ estimated in the 1000 Genomes European reference panels (Nov 2014 EUR). The plot was created using LocusZoom (http://locuszoom.org/).

$D A A M 1$ is a key component of the planar-cell-polarity signaling pathway ${ }^{5,6}$; it acts as a bridging factor between Disheveled, Rho-family GTPases and Rho-associated kinases ${ }^{9}$, a molecular complex involved in organizing actin cytoskeleton ${ }^{10}$.

In order to gain insights into possible mechanisms by which DAAM1 contributes to the tangential expansion of the human visual cortex, we have carried out a number of follow- 
up studies. First, we examined DAAM1 expression in the human brain using the BrainSpan dataset (Table E4 in Extended Data). As shown in Figure 3, DAAM1 is expressed in the cerebral cortex between $\sim 80$ and $\sim 150$ post-conception days; after birth, its expression is very low. Note that, in monkeys, neurogenesis of the primary visual cortex begins around embryonic day 40 (E40) and ends at E100 (165-day gestation) ${ }^{1}$.

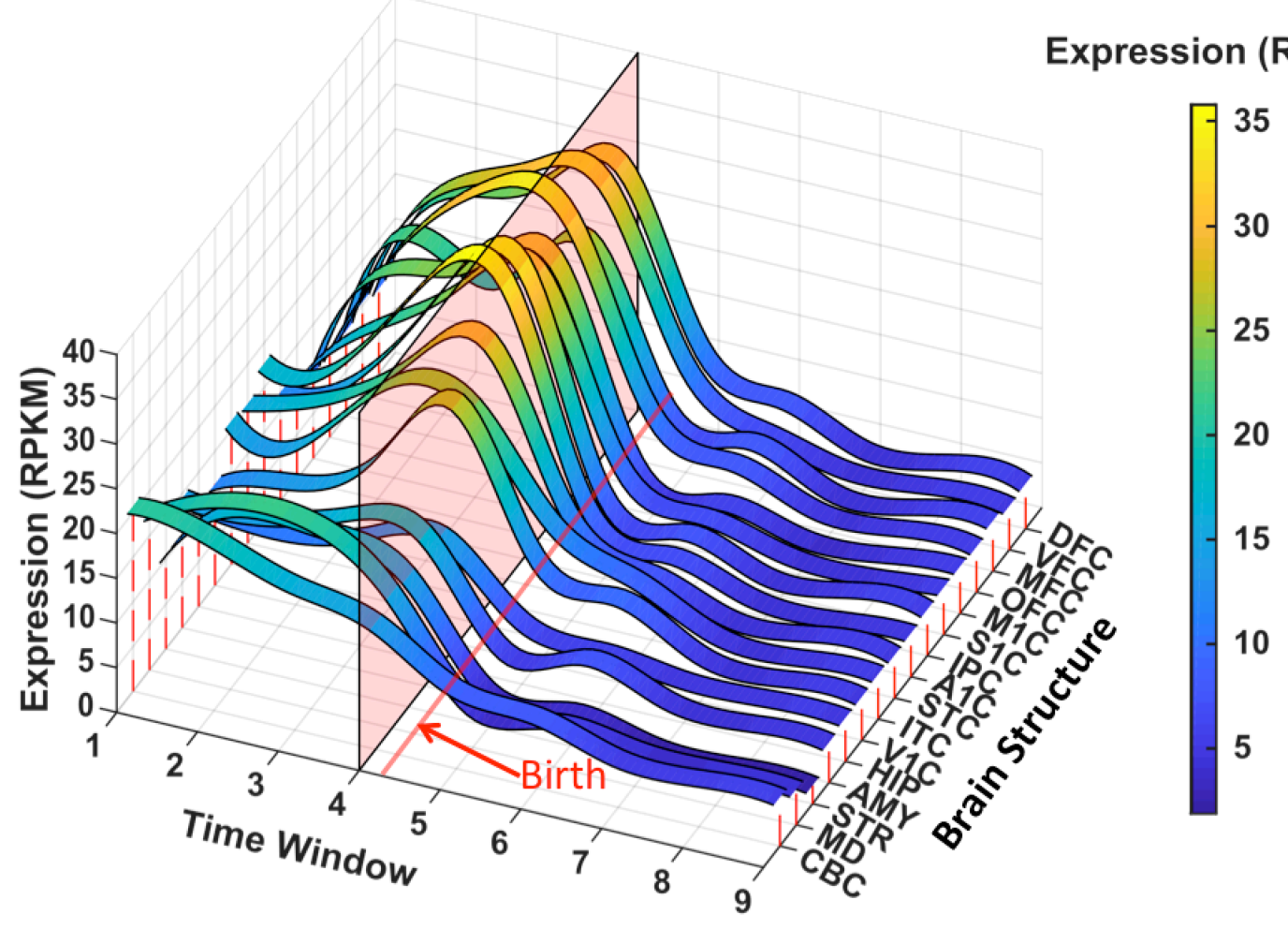

Figure 3. Spatial and temporal expression of DAAM1 on BrainSpan mRNA-seq data. The mRNA expression levels were measured by RNA sequencing in 607 brain tissues from 18 female and 23 male donors available in BrainSpan database (http://www.brainspan.org/). For a given brain structure, DAAM1 expression value was averaged and then fitted across differentiation windows using spline function implemented in MATLAB to get smooth DAAM1 expression dynamics. Each colored band represents the fitted expression levels in RPKM (reads per kilobase per million) of $D A A M 1$. Brain structure includes 11 cortical and 5 sub-cortical regions: dorsolateral prefrontal cortex (DFC), ventrolateral prefrontal cortex (VFC), medial frontal cortex (MFC), orbitofrontal cortex (OFC), primary motor cortex (M1C), primary sensory cortex (S1C), inferior parietal cortex (IPC), primary auditory cortex (A1C), superior temporal cortex (STC), inferior temporal cortex (ITC), primary visual cortex (V1C), hippocampus (HIP), amygdala (AMY), striatum (STR), mediodorsal nucleus of thalamus (MD) and cerebellar cortex (CBC). The human brain differentiation was split into 9 windows based on post conception days: 52-69 (Window 1), 70-111 (Window 2), 112-132 (Window 3), 133-167 (Window 4), 168-447 (Window5), 448 - 1299 (Window 6), 1300-4648 (Window 7), 4649-7570 (Window 8), and 7571-14876 (Window 8). The red line indicates the boundary between pre- and postnatal periods. The pink panel indicates the time where DAAM1 has peak expression in V1C. Blue-to-yellow colors represent low-to-high expression levels of DAAM1 as indicated in the color bar.

Second, we examined co-expression of DAAM1 across all cortical regions and prenatal time points using the same BrainSpan dataset. As expression of DAAM1 increases and decreases, so does expression of genes enriched in pathways involving neuron migration and cytoskeleton organization, among others (Table E5A in Extended Data). 
On the other hand, expression of a large number of genes varies in the direction opposite to that of $D A A M 1$, including genes enriched in pathways involved in a number of metabolic processes (Table E5B in Extended Data). To ascertain the pattern of $D A A M 1$ co-expression specific to the primary visual cortex (V1), we have identified genes co-expressed highly (top 1\%) in V1 but not in any other cortical region (i.e., not present among top $1 \%$ in any of the other eight regions). This analysis yielded striking enrichment for mitochondria-related genes co-expressed strongly in the same direction as DAAM1 in V1 but not in the other cortical regions (Table E6 in Extended Data). This observation turned our attention to the well-known parcellation of the visual cortex to cytochrome-oxidase rich sub-regions, so-called "blobs" (V1) and "stripes" (V2/V3) ${ }^{11}$. We then examined co-localization of DAAM1 and a mitochondrial marker ATP5A in the developing ( $22^{\text {nd }}$ post-conception week) visual cortex (Figure E6); its co-localization is consistent with the co-expression analyses described above. It is known that post-mitotic neurons migrate, along radial glia, from proliferative (ventricular and subventricular) zones to subplate zones, which contain afferents from the thalamic radiation ${ }^{1}$. Activity carried by these afferents from the retina is critical for the development of the primate visual cortex; enucleation of the eyes during fetal development results in the reduction of the surface area (but not thickness) of the monkey primary visual cortex ${ }^{12,13}$, as well as in a reduced size of the lateral geniculate nucleus $(L G N)^{13}$. A tight relationship exists between the volume of LGN and surface area of the primary visual cortex in the human (adult) brain ${ }^{14}$. Furthermore, the koniocellular portion of LGN (which carries signals from short wavelength [blue] cones) appears to project specifically to cytochrome-oxidase rich areas of the visual cortex ${ }^{15}$. Therefore, we hypothesized that DAAM1 contributes to the migration of cytochrome-oxidase positive neurons in response to the LGN inputs in the subplate zones during fetal development. Third, to test this hypothesis in our data, we predicted that the expected relationship between the LGN volume (a proxy for retinal inputs during fetal development) and V1 surface area will be present only in DAAM1 (rs73313052) major-allele homozygotes (GG) but not in the carriers of minor allele (GA or AA). This prediction was confirmed: an interaction between rs73313052 genotype and LGN volume vis-à-vis PC2 magnitude was significant when examined in a cohort with available LGN volumes (GG: $r=0.13, p=0.0006, n=694$; A carriers: $r=-0.06, p=0.37$, $\mathrm{n}=206$; interaction: $\mathrm{t}$ ratio $=-2.47, \mathrm{p}=0.014, \mathrm{n}=925$; Figure $\mathrm{E} 7$ in Extended Data).

In summary, we discovered a non-overlapping set of 99 'independent' single nucleotide polymorphisms within 35 genomic loci contributing to the global and regional tangential growth of the human cerebral cortex (Table E7, in Extended Data). On the other hand, our meta-GWAS of cortical thickness, carried out in the same individuals, yielded only two loci. This negative finding is consistent with a very low number of loci associated with both global and regional values of cortical thickness reported in the two reports from the CHARGE $^{16}$ and ENIGMA ${ }^{17}$ Consortia; it may reflect substantial dynamics of cortical thickness during puberty ${ }^{18}$ and aging ${ }^{19}$. For surface area PC1 (PC2), we replicated 695/ 807 (PC1) and 952/1,155 (PC2) GWAS-significant SNPs (Table E2A and E2B, in Extended Data). Through a series of follow-up studies, we formulated a working model by which $D A A M 1$ regulates tangential expansion of the visual cortex by interacting with LGN inputs, likely at the level of cortical subplate, during mid-gestation. A larger visual cortex is likely to posses more inter-hemispheric connections; DAAM1 polymorphism is associated with a structure-predicted functional connectivity of the human visual cortex ${ }^{20}$. Overall, these findings illustrate how specification of cortical areas, and their relative growth, might be guided by an interaction between fetal environment and generic developmental mechanisms, such as those constituting planar-cell-polarity signaling pathway. 


\section{Acknowledgements}

This research has been conducted using the UK Biobank Resource under Application Number ' 23509'.

\section{References}

1. Rakic P. Specification of cerebral cortical areas. Science. Jul 8 1988;241(4862):170-176.

2. Adams HH, Hibar DP, Chouraki V, et al. Novel genetic loci underlying human intracranial volume identified through genome-wide association. Nat Neurosci. Oct 032016.

3. Hibar DP, Stein JL, Renteria ME, et al. Common genetic variants influence human subcortical brain structures. Nature. Apr 9 2015;520(7546):224-229.

4. Trampush JW, Yang ML, Yu J, et al. GWAS meta-analysis reveals novel loci and genetic correlates for general cognitive function: a report from the COGENT consortium. Mol Psychiatry. Mar 2017;22(3):336-345.

5. Tissir F, Goffinet AM. Planar cell polarity signaling in neural development. Curr Opin Neurobiol. Oct 2010;20(5):572-577.

6. Beane WS, Tseng AS, Morokuma J, Lemire JM, Levin M. Inhibition of planar cell polarity extends neural growth during regeneration, homeostasis, and development. Stem cells and development. Aug 10 2012;21(12):2085-2094.

7. Selemon LD, Ceritoglu C, Ratnanather JT, et al. Distinct abnormalities of the primate prefrontal cortex caused by ionizing radiation in early or midgestation. J Comp Neurol. Apr 1 2013;521(5):1040-1053.

8. Nieuwboer HA, Pool R, Dolan CV, Boomsma DI, Nivard MG. GWIS: GenomeWide Inferred Statistics for Functions of Multiple Phenotypes. Am J Hum Genet. Oct 6 2016;99(4):917-927.

9. Habas R, Kato Y, He X. Wnt/Frizzled activation of Rho regulates vertebrate gastrulation and requires a novel Formin homology protein Daam1. Cell. Dec 28 2001;107(7):843-854.

10. Yang Y, Mlodzik M. Wnt-Frizzled/planar cell polarity signaling: cellular orientation by facing the wind (Wnt). Annual review of cell and developmental biology. 2015;31:623-646.

11. Livingstone MS, Hubel DH. Thalamic inputs to cytochrome oxidase-rich regions in monkey visual cortex. Proc Natl Acad Sci $U S A$. Oct 1982;79(19):6098-6101.

12. Bourgeois JP, Rakic P. Synaptogenesis in the occipital cortex of macaque monkey devoid of retinal input from early embryonic stages. Eur J Neurosci. May 1996;8(5):942-950.

13. Dehay C, Giroud P, Berland M, Killackey H, Kennedy H. Contribution of thalamic input to the specification of cytoarchitectonic cortical fields in the primate: effects of bilateral enucleation in the fetal monkey on the 
boundaries, dimensions, and gyrification of striate and extrastriate cortex. $J$ Comp Neurol. Mar 25 1996;367(1):70-89.

14. Andrews TJ, Halpern SD, Purves D. Correlated size variations in human visual cortex, lateral geniculate nucleus, and optic tract. J Neurosci. Apr 15 1997;17(8):2859-2868.

15. Hendry SH, Reid RC. The koniocellular pathway in primate vision. Annu Rev Neurosci. 2000;23:127-153.

16. Hofer Eac. Genetic Determinants of Cortical Structure (Thickness, Surface Area and Volumes) among Disease Free Adults in the CHARGE Consortium. BioRxiv2018.

17. Grasby Kac. The genetic architecture of the human cerebral cortex. BioRxiv2018.

18. Walhovd KB, Fjell AM, Giedd J, Dale AM, Brown TT. Through Thick and Thin: a Need to Reconcile Contradictory Results on Trajectories in Human Cortical Development. Cereb Cortex. Feb 01 2017;27(2):1472-1481.

19. Vinke EJ, de Groot $M$, Venkatraghavan $V$, et al. Trajectories of imaging markers in brain aging: the Rotterdam Study. Neurobiol Aging. Jul 17 2018;71:32-40.

20. Mollink J, Smith SM, Elliott LT, et al. The spatial correspondence and genetic influence of interhemispheric connectivity with white matter microstructure. Nat Neurosci. Apr 152019.

\section{ENIGMA Consortium Authors}

Katrina L. Grasby (Psychiatric Genetics, QIMR Berghofer Medical Research Institute, Brisbane, Australia), Neda Jahanshad (Imaging Genetics Center, Mark and Mary Stevens Neuroimaging and Informatics Institute, Keck School of Medicine of USC, University of Southern California, Los Angeles, USA), Jodie N. Painter (Psychiatric Genetics, QIMR Berghofer Medical Research Institute, Brisbane, Australia), Lucía Colodro-Conde (Psychiatric Genetics, QIMR Berghofer Medical Research Institute, Brisbane, Australia), Janita Bralten (Department of Human Genetics, Radboud university medical center, Nijmegen, The Netherlands), Derrek P. Hibar (Imaging Genetics Center, Mark and Mary Stevens Neuroimaging and Informatics Institute, Keck School of Medicine of USC, University of Southern California, Los Angeles, USA), Penelope A. Lind (Psychiatric Genetics, QIMR Berghofer Medical Research Institute, Brisbane, Australia), Fabrizio Pizzagalli (Imaging Genetics Center, Mark and Mary Stevens Neuroimaging and Informatics Institute, Keck School of Medicine of USC, University of Southern California, Los Angeles, USA), Christopher R.K. Ching (Imaging Genetics Center, Mark and Mary Stevens Neuroimaging and Informatics Institute, Keck School of Medicine of USC, University of Southern California, Los Angeles, USA), Mary Agnes B. McMahon (Imaging Genetics Center, Mark and Mary Stevens Neuroimaging and Informatics Institute, Keck School of Medicine of USC, University of Southern California, Los Angeles, USA), Natalia Shatokhina (Imaging Genetics Center, Mark and Mary Stevens Neuroimaging and Informatics Institute, Keck School of Medicine of USC, University of Southern California, Los Angeles, USA), Leo Zsembik (Department of Genetics \& UNC Neuroscience Center, University of North Carolina at Chapel HIII, Chapel Hill, USA), Ingrid Agartz (NORMENT - K.G. Jebsen Centre for Psychosis 
Research, Division of Mental Health and Addiction, NORMENT Oslo University Hospital, Oslo, Norway), Saud Alhusaini (Department of Molecular and Cellular Therapeutics, Royal College of Surgeons in Ireland, Dublin, Ireland), Marcio A.A. Almeida (Department of Human Genetics and South Texas Diabetes and Obesity Institute, Rio Grande Valley School of Medicine, University of Texas, Brownsville, USA), Dag Alnæs (NORMENT K.G. Jebsen Centre for Psychosis Research, Division of Mental Health and Addiction, NORMENT Oslo University Hospital, Oslo, Norway), Inge K. Amlien (Centre for Lifespan Changes in Brain and Cognition, Department of Psychology, University of Oslo, Oslo, Norway), Micael Andersson (Department of Integrative Medical Biology, Umeå University, Umeå, Sweden), Tyler Ard (Laboratory of Neuro Imaging, Mark and Mary Stevens Neuroimaging and Informatics Institute, Keck School of Medicine, University of Southern California, Los Angeles, USA), Nicola J. Armstrong (Mathematics and Statistics, Murdoch University, Murdoch, Australia), Allison Ashley-Koch (Duke Molecular Physiology Institute, Duke University Medical Center, Durham, USA), Manon Bernard (The Hospital for Sick Children, University of Toronto, Toronto, Canada), Rachel M. Brouwer (Department of Psychiatry, Brain Center Rudolf Magnus, University Medical Center Utrecht, Utrecht University, Utrecht, The Netherlands), Elizabeth E.L. Buimer (Department of Psychiatry, Brain Center Rudolf Magnus, University Medical Center Utrecht, Utrecht University, Utrecht, The Netherlands), Robin Bülow (Institute for Radiology and Neuroradiology, University Medicine, Ernst-Moritz-Arndt University, Greifswald, Germany), Christian Bürger (Department of Psychiatry, University of Münster, Münster, Germany), Dara M. Cannon (Centre for Neuroimaging \& Cognitive Genomics, National University of Ireland Galway, Galway, Ireland), Mallasr Chakravarty (Douglas Mental Health University Institute, McGill University, Montreal, Canada), Qiang Chen (Lieber Institute for Brain Development, Baltimore, USA), Joshua W. Cheung (Imaging Genetics Center, Mark and Mary Stevens Neuroimaging and Informatics Institute, Keck School of Medicine of USC, University of Southern California, Los Angeles, USA), Baptiste Couvy-Duchesne (Institute for Molecular Bioscience, The University of Queensland, Brisbane, Australia), Anders M. Dale (Department of Neurosciences, University of California, San Diego, La Jolla), Shareefa Dalvie (Department of Psychiatry and Mental Health, University of Cape Town, Cape Town, South Africa), Tânia K. de Araujo (Department of Medical Genetics, School of Medical Sciences, University of Campinas - UNICAMP, Campinas, Brazil), Greig I. de Zubicaray (Faculty of Health, Institute of Health and Biomedical Innovation, Queensland University of Technology, Brisbane, Australia), Sonja M.C. de Zwarte (Department of Psychiatry, Brain Center Rudolf Magnus, University Medical Center Utrecht, Utrecht University, Utrecht, The Netherlands), Anouk den Braber (Department of Biological Psychology, Vrije Universiteit Amsterdam, Amsterdam, The Netherlands), Nhat Trung Doan (NORMENT - K.G. Jebsen Centre for Psychosis Research, Division of Mental Health and Addiction, NORMENT Oslo University Hospital, Oslo, Norway), Katharina Dohm (Department of Psychiatry, University of Münster, Münster, Germany), Stefan Ehrlich (Division of Psychological \& Social Medicine and Developmental Neurosciences, Technische Universität Dresden, Dresden, Germany), Hannah-Ruth Engelbrecht (Division of Human Genetics, Institute of Infectious Disease and Molecular Medicine, University of Cape Town, Cape Town, South Africa), Susanne Erk (Division of Mind and Brain Research, Department of Psychiatry and Psychotherapy, Campus Charite Mitte, Charité - Universitätsmedizin Berlin, Berlin, Germany), Chun Chieh Fan (Department of Cognitive Science, University of California San Diego, San Diego, USA), Iryna O. Fedko (Department of Biological Psychology, Vrije Universiteit Amsterdam, Amsterdam, The Netherlands), Sonya F. Foley (Cardiff University Brain Research Imaging Centre, Cardiff University, Cardiff, UK), Judith M. Ford (San Francisco Veterans Administration Medical 
Center, San Francisco, USA), Masaki Fukunaga (Division of Cerebral Integration, National Institute for Physiological Sciences, Okazaki, Japan), Melanie E. Garrett (Duke Molecular Physiology Institute, Duke University Medical Center, Durham, USA), Tian Ge (Psychiatric and Neurodevelopmental Genetics Unit, Center for Genomic Medicine, Massachusetts General Hospital, Boston, USA), Sudheer Giddaluru (NORMENT - K.G. Jebsen Centre for Psychosis Research, Department of Clinical Science, NORMENT University of Bergen, Bergeb, Norway), Aaron L. Goldman (Lieber Institute for Brain Development, Baltimore, USA), Nynke A. Groenewold (Department of Psychiatry and Mental Health, University of Cape Town, Cape Town, South Africa), Dominik Grotegerd (Department of Psychiatry, University of Münster, Münster, Germany), Tiril P. Gurholt (NORMENT - K.G. Jebsen Centre for Psychosis Research, Division of Mental Health and Addiction, NORMENT Oslo University Hospital, Oslo, Norway), Boris A. Gutman (Imaging Genetics Center, Mark and Mary Stevens Neuroimaging and Informatics Institute, Keck School of Medicine of USC, University of Southern California, Los Angeles, USA), Narelle K. Hansell (Queensland Brain Institute, University of Queensland, St Lucia, Australia), Mathew A. Harris (Centre for Clinical Brain Sciences and Edinburgh Imaging, University of Edinburgh, Edinburgh, UK), Marc B. Harrison (Imaging Genetics Center, Mark and Mary Stevens Neuroimaging and Informatics Institute, Keck School of Medicine of USC, University of Southern California, Los Angeles, USA), Courtney C. Haswell (Duke UNC Brain Imaging and Analysis Center, Duke University Medical Center, Durham, USA), Michael Hauser (Duke Molecular Physiology Institute, Duke University Medical Center, Durham, USA), Dirk J. Heslenfeld (Department of Cognitive and Clinical Neuropsychology, Vrije Universiteit Amsterdam, Amsterdam, The Netherlands), David Hoehn (Max Planck Institute of Psychiatry, Munich, Germany), Laurena Holleran (Centre for Neuroimaging \& Cognitive Genomics, National University of Ireland Galway, Galway, Ireland), Martine Hoogman (Department of Human Genetics, Radboud university medical center, Nijmegen, The Netherlands), Jouke-Jan Hottenga (Department of Biological Psychology, Vrije Universiteit Amsterdam, Amsterdam, The Netherlands), Masashi Ikeda (Department of Psychiatry, Fujita Health University School of Medicine, Toyoake, Japan), Deborah Janowitz (Department of Psychiatry and Psychotherapy, University Medicine Greifswald, Greifswald, Germany), Iris E. Jansen (Department of Complex Trait Genetics, Vrije Universiteit Amsterdam, Amsterdam, The Netherlands), Tianye Jia (Institute of Science and Technology for Brain-Inspired Intelligence, Fudan University, Shanghai, China), Christiane Jockwitz (Institute of Neuroscience and Medicine (INM-1), Research Centre Jülich, Jülich, Germany), Ryota Kanai (Department of Neuroinformatics, Araya, Inc., Inc. , Tokyo), Sherif Karama (Department of Psychiatry, McGill University, Montreal, Canada), Dalia Kasperaviciute (Department of Clinical and Experimental Epilepsy, University College London, London, UK), Tobias Kaufmann (NORMENT - K.G. Jebsen Centre for Psychosis Research, Division of Mental Health and Addiction, NORMENT Oslo University Hospital, Oslo, Norway), Sinead Kelly (Public Psychiatry Division, Massachusetts Mental Health Center, Beth Israel Deaconess Medical Center, Harvard Medical School, Boston, USA), Masataka Kikuchi (Department of Genome Informatics, Graduate School of Medicine, Osaka University, Suita, Japan), Marieke Klein (Department of Human Genetics, Radboud university medical center, Nijmegen, The Netherlands), Michael Knapp (Department of Medical Biometry, Informatics and Epidemiology, University Hospital Bonn, Bonn, Germany), Annchen R. Knodt (Department of Psychology and Neuroscience, Duke University, Durham, USA), Bernd Krämer (Section for Experimental Psychopathology and Neuroimaging, Department of General Psychiatry, Heidelberg University Hospital, Heidelberg, Germany), Thomas M. Lancaster (Cardiff University Brain Research Imaging Centre, Cardiff University, Cardiff, UK), Phil H. Lee (Psychiatric and Neurodevelopmental 
Genetics Unit, Center for Genomic Medicine, Massachusetts General Hospital, Boston, USA), Tristram A. Lett (Division of Mind and Brain Research, Department of Psychiatry and Psychotherapy, Campus Charité Mitte, Charité - Universitätsmedizin Berlin, Berlin, Germany), Lindsay B. Lewis (McGill Centre for Integrative Neuroscience, McGill University, Montreal, Canada), Iscia Lopes-Cendes (Department of Medical Genetics, School of Medical Sciences, University of Campinas - UNICAMP, Campinas, Brazil), Michelle Luciano (Department of Psychology, University of Edinburgh, Edinburgh, UK), Fabio Macciardi (Department of Psychiatry and Human Behavior, School of Medicine , University of California, Irvine, Irvine, USA), Andre F. Marquand (Department of Cognitive Neuroscience, Radboud university medical center, Nijmegen, The Netherlands), Samuel R. Mathias (Department of Psychiatry, Yale University School of Medicine, New Haven, USA), Tracy R. Melzer (Department of Medicine, University of Otago, Christchurch, Christchurch, New Zealand), Yuri Milaneschi (Psychiatry, Amsterdam UMC Vrije Universiteit, Psychiatry, The Netherlands), Nazanin MirzaSchreiber (Max Planck Institute of Psychiatry, Munich, Germany), Jose C.V. Moreira (, BRAINN - Brazilian Institute of Neuroscience and Neurotechnology, Campinas, Brasil), Thomas W. Mühleisen (Institute of Neuroscience and Medicine (INM-1), Research Centre Jülich, Jülich, Germany), Bertram Müller-Myhsok (Max Planck Institute of Psychiatry, Munich, Germany), Pablo Najt (Centre for Neuroimaging \& Cognitive Genomics, National University of Ireland Galway, Galway, Ireland), Soichiro Nakahara (Department of Psychiatry and Human Behavior, School of Medicine University of California, University of California, Irvine, Irvine, USA), Kwangsik Nho (Department of Radiology and Imaging Sciences, Indiana University School of Medicine, Indianapolis, USA), Loes M. Olde Loohuis (Center for Neurobehavioral Genetics, University of California Los Angeles, Los Angeles, USA), Dimitri Papadopoulos Orfanos (NeuroSpin, CEA, Université Paris-Saclay, Gif sur Yvette, France), John F. Pearson (Biostatistics and Computational Biology Unit, University of Otago, Christchurch, Christchurch, New Zealand), Toni L. Pitcher (Department of Medicine, University of Otago, Christchurch, Christchurch, New Zealand), Benno Pütz (Max Planck Institute of Psychiatry, Munich, Germany), Anjanibhargavi Ragothaman (Imaging Genetics Center, Mark and Mary Stevens Neuroimaging and Informatics Institute, Keck School of Medicine of USC, University of Southern California, Los Angeles, USA), Faisal M. Rashid (Imaging Genetics Center, Mark and Mary Stevens Neuroimaging and Informatics Institute, Keck School of Medicine of USC, University of Southern California, Los Angeles, USA), Ronny Redlich (Department of Psychiatry, University of Münster, Münster, Germany), Céline S. Reinbold (Department of Biomedicine, University of Basel, Basel, Switzerland), Jonathan Repple (Department of Psychiatry, University of Münster, Münster, Germany), Geneviève Richard (NORMENT - K.G. Jebsen Centre for Psychosis Research, Division of Mental Health and Addiction, NORMENT Oslo University Hospital, Oslo, Norway), Brandalyn C. Riedel (Imaging Genetics Center, Mark and Mary Stevens Neuroimaging and Informatics Institute, Keck School of Medicine of USC, University of Southern California, Los Angeles, USA), Shannon L. Risacher (Department of Radiology and Imaging Sciences, Indiana University School of Medicine, Indianapolis, USA), Cristiane S. Rocha (Department of Medical Genetics, School of Medical Sciences, University of Campinas - UNICAMP, Campinas, Brazil), Nina Roth Mota (Department of Human Genetics, Radboud university medical center, Nijmegen, The Netherlands), Lauren Salminen (Imaging Genetics Center, Mark and Mary Stevens Neuroimaging and Informatics Institute, Keck School of Medicine of USC, University of Southern California, Los Angeles, USA), Arvin Saremi (Imaging Genetics Center, Mark and Mary Stevens Neuroimaging and Informatics Institute, Keck School of Medicine of USC, University of Southern California, Los Angeles, USA), Andrew J. Saykin (Department of Radiology 
and Imaging Sciences, Indiana University School of Medicine, Indianapolis, USA), Fenja Schlag (Language and Genetics Department, Max Planck Institute for Psycholinguistics, Nijmegen, The Netherlands), Lianne Schmaal (Orygen, The National Centre of Excellence for Youth Mental Health, Melbourne, Australia), Peter R. Schofield (Neuroscience Research Australia, Sydney, Australia), Rodrigo Secolin (Department of Medical Genetics, School of Medical Sciences, University of Campinas - UNICAMP, Campinas, Brazil), Chin Yang Shapland (Language and Genetics Department, Max Planck Institute for Psycholinguistics, Nijmegen, The Netherlands), Li Shen (Department of Biostatistics, Epidemiology and Informatics, University of Pennsylvania, Philadelphia, USA), Jean Shin (The Hospital for Sick Children, University of Toronto, Toronto, Canada), Elena Shumskaya (Department of Human Genetics, Radboud university medical center, Nijmegen, The Netherlands), Ida E. Sønderby (NORMENT - K.G. Jebsen Centre for Psychosis Research, Division of Mental Health and Addiction, NORMENT Oslo University Hospital, Oslo, Norway), Emma Sprooten (Donders Institute for Brain, Cognition and Behaviour, Radboud University, Nijmegen, The Netherlands), Lachlan T. Strike (Queensland Brain Institute, University of Queensland, St Lucia, Australia), Katherine E. Tansey (MRC Centre for Neuropsychiatric Genetics and Genomics, Cardiff University, Cardiff, UK), Alexander Teumer (Institute for Community Medicine, University Medicine Greifswald, Greifswald, Germany), Anbupalam Thalamuthu (Centre for Healthy Brain Ageing, University of New South Wales, Sydney, Australia), Sophia I. Thomopoulos (Imaging Genetics Center, Mark and Mary Stevens Neuroimaging and Informatics Institute, Keck School of Medicine of USC, University of Southern California, Los Angeles, USA), Diana Tordesillas-Gutiérrez (Neuroimaging Unit, Valdecilla Biomedical Research Institute IDIVAL, Santander, Spain), Jessica A. Turner (Department of Psychology, Georgia State University, Atlanta, USA), Anne UhImann (Department of Psychiatry and Mental Health, University of Cape Town, Cape Town, South Africa), Costanza Ludovica Vallerga (Institute for Molecular Bioscience, The University of Queensland, Brisbane, Australia), Dennis van der Meer (NORMENT - K.G. Jebsen Centre for Psychosis Research, Division of Mental Health and Addiction, NORMENT Oslo University Hospital, Oslo, Norway), Marjolein M.J. van Donkelaar (Department of Human Genetics, Radboud university medical center, Nijmegen, The Netherlands), Liza van Eijk (School of Psychology, University of Queensland, Brisbane, Australia), Theo G.M. van Erp (Department of Psychiatry and Human Behavior, School of Medicine University of California, University of California, Irvine, Irvine, USA), Neeltje E.M. van Haren (Department of Psychiatry, Brain Center Rudolf Magnus, University Medical Center Utrecht, Utrecht University, Utrecht, The Netherlands), Daan van Rooij (Department of Cognitive Neuroscience, Radboud university medical center, Nijmegen, The Netherlands), Marie-José van Tol (Cognitive Neuroscience Center, Department of Neuroscience, Cardiff University, Groningen, The Netherlands), Jan H. Veldink (Department of Neurology, Brain Center Rudolf Magnus, University Medical Center Utrecht, Utrecht University, Utrecht, The Netherlands), Ellen Verhoef (Language and Genetics Department, Max Planck Institute for Psycholinguistics, Nijmegen, The Netherlands), Esther Walton (Department of Psychology, Georgia State University, Atlanta, USA), Yunpeng Wang (NORMENT - K.G. Jebsen Centre for Psychosis Research, Division of Mental Health and Addiction, NORMENT Oslo University Hospital, Oslo, Norway), Joanna M. Wardlaw (Centre for Clinical Brain Sciences and Edinburgh Imaging, University of Edinburgh, Edinburgh, UK), Wei Wen (Centre for Healthy Brain Ageing, University of New South Wales, Sydney, Australia), Lars T. Westlye (NORMENT - K.G. Jebsen Centre for Psychosis Research, Division of Mental Health and Addiction, NORMENT Oslo University Hospital, Oslo, Norway), Christopher D. Whelan (Imaging Genetics Center, Mark and Mary Stevens Neuroimaging and Informatics Institute, Keck 
School of Medicine of USC, University of Southern California, Los Angeles, USA), Stephanie H. Witt (Department of Genetic Epidemiology in Psychiatry, Central Institute of Mental Health, Medical Faculty Mannheim, Heidelberg University, Mannheim, Germany), Katharina Wittfeld (German Center for Neurodegenerative Diseases Rostock/Greifswald, Greifswald, Germany), Christiane Wolf (Department of Psychiatry, Psychosomatics and Psychotherapy, University of Würzburg, Würzburg, Germany), Thomas Wolfers (Department of Human Genetics, Radboud university medical center, Nijmegen, The Netherlands), Clarissa L. Yasuda (Department of Neurology, FCM, University of Campinas - UNICAMP, Campinas, Brasil), Dario Zaremba (Department of Psychiatry, University of Münster, Münster, Germany), Zuo Zhang (Social, Genetic and Developmental Psychiatry Centre, Institute of Psychiatry, Psychology \& Neuroscience, King's College London, London, UK), Alyssa H. Zhu (Imaging Genetics Center, Mark and Mary Stevens Neuroimaging and Informatics Institute, Keck School of Medicine of USC, University of Southern California, Los Angeles, USA), Marcel P. Zwiers (Department of Cognitive Neuroscience, Radboud university medical center, Nijmegen, The Netherlands), Eric Artiges (INSERM Unit 1000 - Neuroimaging \& Psychiatry, Paris Sacaly University, Gif sur Yvette, France), Amelia A. Assareh (Centre for Healthy Brain Ageing, University of New South Wales, Sydney, Australia), Rosa Ayesa-Arriola (Department of Psychiatry, University Hospital Marqués de Valdecilla, School of Medicine, University of Cantabria-IDIVAL, Santander, Spain), Aysenil Belger (Duke UNC Brain Imaging and Analysis Center, Duke University Medical Center, Durham, USA), Christine L. Brandt (NORMENT - K.G. Jebsen Centre for Psychosis Research, Division of Mental Health and Addiction, NORMENT Oslo University Hospital, Oslo, Norway), Gregory G. Brown (Department of Psychiatry, University of California San Diego, San Diego, USA), Sven Cichon (Department of Biomedicine, University of Basel, Basel, Switzerland), Joanne E. Curran (Department of Human Genetics and South Texas Diabetes and Obesity Institute, Rio Grande Valley School of Medicine, University of Texas, Brownsville, USA), Gareth E. Davies (Avera Institute for Human Genetics, Sioux Falls, USA), Franziska Degenhardt (Institute of Human Genetics, School of Medicine \& University Hospital Bonn, University of Bonn, Bonn, Germany), Bruno Dietsche (Department of Psychiatry and Psychotherapy, Philipps-University Marburg, Marburg, Germany), Srdjan Djurovic (Department of Medical Genetics, Oslo University Hospital, Oslo, Norway), Colin P. Doherty (Department of Neurology, St James's Hospital, Dublin, Ireland), Ryan Espiritu (Information Sciences Institute, University of Southern California, Los Angeles, USA), Daniel Garijo (Information Sciences Institute, University of Southern California, Los Angeles, USA), Yolanda Gil (Information Sciences Institute, University of Southern California, Los Angeles, USA), Penny A. Gowland (Sir Peter Mansfield Imaging Centre, University of Nottingham, Nottingham, UK), Robert C. Green (Brigham and Women's Hospital, Boston, USA), Alexander N. Häusler (Center for Economics and Neuroscience, University of Bonn, Bonn, Germany), Walter Heindel (Department of Clinical Radiology, University of Münster, Münster, Germany), BengChoon Ho (Department of Psychiatry, University of lowa College of Medicine, lowa City, USA), Wolfgang U. Hoffmann (Institute for Community Medicine, University Medicine Greifswald, Greifswald, Germany), Florian Holsboer (HMNC Holding GmbH, Munich, Germany), Georg Homuth (Department of Functional Genomics, Interfaculty Institute for Genetics and Functional Genomics, University Medicine Greifswald, Greifswald, Germany), Norbert Hosten (Institute of Diagnostic Radiology and Neuroradiology, Greifswald, Germany), Clifford R. Jack Jr. (Dept of Radiology, Mayo Clinic, Rochester, USA), MiHyun Jang (Information Sciences Institute, University of Southern California, Los Angeles, USA), Andreas Jansen (Department of Psychiatry and Psychotherapy, Philipps-University Marburg, Marburg, Germany), Knut Kolskår (NORMENT - K.G. 
Jebsen Centre for Psychosis Research, Division of Mental Health and Addiction, NORMENT Oslo University Hospital, Oslo, Norway), Sanne Koops (Department of Psychiatry, Brain Center Rudolf Magnus, University Medical Center Utrecht, Utrecht University, Utrecht, The Netherlands), Axel Krug (Department of Psychiatry and Psychotherapy, Philipps-University Marburg, Marburg, Germany), Kelvin O. Lim (Department of Psychiatry, University of Minnesota, Minneapolis, USA), Jurjen J. Luykx (Department of Translational Neuroscience, Brain Center Rudolf Magnus, University Medical Center Utrecht, Utrecht University, Utrecht, The Netherlands), Daniel H. Mathalon (Department of Psychiatry and Weill Institute for Neurosciences, University of California San Francisco, San Francisco, USA), Karen A. Mather (Centre for Healthy Brain Ageing, University of New South Wales, Sydney, Australia), Venkata S. Mattay (Lieber Institute for Brain Development, Baltimore, USA), Sarah Matthews (MRC Integrative Epidemiology Unit, Department of Population Health Sciences, Bristol Medical School, Bristol, UK), Jaqueline Mayoral Van Son (Department of Psychiatry, University Hospital Marqués de Valdecilla, School of Medicine, University of CantabriaIDIVAL, Santander, Spain), Sarah C. McEwen (Department of Psychiatry, University of California San Diego, La Jolla, USA), Ingrid Melle (NORMENT - K.G. Jebsen Centre for Psychosis Research, Division of Mental Health and Addiction, NORMENT Oslo University Hospital, Oslo, Norway), Derek W. Morris (Centre for Neuroimaging \& Cognitive Genomics, National University of Ireland Galway, Galway, Ireland), Bryon A. Mueller (Department of Psychiatry, University of Minnesota, Minneapolis, USA), Matthias Nauck (Institute of Clinical Chemistry and Laboratory Medicine, University Medicine Greifswald, Greifswald, Germany), Jan E. Nordvik (Sunnaas Rehabilitation Hospital HT, Nesodden, Norway), Markus M. Nöthen (Institute of Human Genetics, School of Medicine \& University Hospital Bonn, University of Bonn, Bonn, Germany), Daniel S. O'Leary (Department of Psychiatry, University of lowa College of Medicine, lowa City, USA), Nils Opel (Department of Psychiatry, University of Münster, Münster, Germany), Marie - Laure Paillère Martinot (INSERM Unit 1000 - Neuroimaging \& Psychiatry, Paris Sacaly University, Gif sur Yvette, France), G. Bruce Pike (Radiology and Clinical Neurosciences, Hotchkiss Brain Institute, University of Calgary, Calgary, Canada), Adrian Preda (School of Medicine, University of California Irvine, Irvine, USA), Erin B. Quinlan (Social, Genetic and Developmental Psychiatry Centre, King's College London, London, UK), Varun Ratnakar (Information Sciences Institute, University of Southern California, Los Angeles, USA), Simone Reppermund (Centre for Healthy Brain Ageing, University of New South Wales, Sydney, Australia), Vidar M. Steen (NORMENT - K.G. Jebsen Centre for Psychosis Research, Department of Clinical Science, NORMENT University of Bergen, Bergen, Norway), Fábio R. Torres (Department of Medical Genetics, School of Medical Sciences, University of Campinas - UNICAMP, Campinas, Brazil), Dick J. Veltman (Psychiatry, Amsterdam UMC Vrije Universiteit, Psychiatry, The Netherlands), James T. Voyvodic (Duke UNC Brain Imaging and Analysis Center, Duke University Medical Center, Durham, USA), Robert Whelan (School of Psychology, Trinity College Dublin, Dublin, Ireland), Tonya White (Department of Child and Adolescent Psychiatry/Psychology, Erasmus Medical Center-Sophia Children's Hospital, Rotterdam, The Netherlands), Hidenaga Yamamori (Department of Psychiatry, Osaka University Graduate School of Medicine, Suita, Japan), Marina K.M. Alvim (Department of Neurology, FCM, University of Campinas - UNICAMP, Campinas, Brasil), David Ames (Academic Unit for Psychiatry of Old Age, University of Melbourne, Melbourne, Australia), Tim J. Anderson (Department of Medicine, University of Otago, Christchurch, Christchurch, New Zealand), Ole A. Andreassen (NORMENT - K.G. Jebsen Centre for Psychosis Research, Division of Mental Health and Addiction, NORMENT Oslo University Hospital, Oslo, Norway), Alejandro Arias-Vasquez (Department of Psychiatry, 
Radboud university medical center, Nijmegen, The Netherlands), Mark E. Bastin (Centre for Clinical Brain Sciences and Edinburgh Imaging, University of Edinburgh, Edinburgh, UK), Bernhard T. Baune (Department of Psychiatry, The University of Melbourne, Melbourne, Australia.), John Blangero (Department of Human Genetics and South Texas Diabetes and Obesity Institute, Rio Grande Valley School of Medicine, University of Texas, Brownsville, USA), Dorret I. Boomsma (Department of Biological Psychology, Vrije Universiteit Amsterdam, Amsterdam, The Netherlands), Henry Brodaty (Centre for Healthy Brain Ageing, University of New South Wales, Sydney, Australia), Han G.

Brunner (Department of Human Genetics, Radboud university medical center, Nijmegen, The Netherlands), Randy L. Buckner (Department of Psychology and Center for Brain Science, Harvard University, Boston, USA), Jan K. Buitelaar (Department of Cognitive Neuroscience, Radboud university medical center, Nijmegen, The Netherlands), Juan R. Bustillo (Department of Psychiatry, University of New Mexico, Albuquerque, USA), Wiepke Cahn (Department of Psychiatry, University Medical Center Utrecht, Utrecht University, Utrecht, The Netherlands), Vince Calhoun (Department of Electrical and Computer Engineering, The University of New Mexico, Albuquerque, USA), Xavier Caseras (MRC Centre for Neuropsychiatric Genetics and Genomics, Cardiff University, Cardiff, UK), Svenja Caspers (Institute for Anatomy I Medical Faculty, Heinrich-Heine University, Düsseldorf, Germany), Gianpiero L. Cavalleri (Molecular and Cellular Therapeutics, The Royal College of Surgeons In Ireland, Dublin, Ireland), Fernando Cendes (Department of Neurology, FCM, University of Campinas - UNICAMP, Campinas, Brasil), Aiden Corvin (Department of Psychiatry, School of Medicine, Trinity College Dublin, Dublin, Ireland), Benedicto Crespo-Facorro (Department of Psychiatry, University Hospital Marqués de Valdecilla, School of Medicine, University of CantabriaIDIVAL, Santander, Spain), John C. Dalrymple-Alford (Department of Psychology, University of Canterbury, Christchurch, New Zealand), Udo Dannlowski (Department of Psychiatry, University of Münster, Münster, Germany), Eco J.C. de Geus (Department of Biological Psychology, Vrije Universiteit Amsterdam, Amsterdam, The Netherlands), Ian J. Deary (Centre for Cognitive Ageing and Cognitive Epidemiology, University of Edinburgh, Edinburgh, UK), Norman Delanty (FutureNeuro Research Centre, Royal College of Surgeons in Ireland, Dublin, Ireland), Chantal Depondt (Department of Neurology, Hôpital Erasme, Université Libre de Bruxelles, Brussels, Belgium), Sylvane Desrivières (Social, Genetic and Developmental Psychiatry Centre, King's College London, London, UK), Gary Donohoe (Centre for Neuroimaging \& Cognitive Genomics, National University of Ireland Galway, Galway, Ireland), Thomas Espeseth (Department of Psychology, University of Oslo, Oslo, Norway), Guillén Fernández (Department of Cognitive Neuroscience, Radboud university medical center, Nijmegen, The Netherlands), Simon E. Fisher (Language and Genetics Department, Max Planck Institute for Psycholinguistics, Nijmegen, The Netherlands), Herta Flor (Department of Cognitive and Clinical Neuroscience, Central Institute of Mental Health, Medical Faculty Mannheim, Heidelberg University, Mannheim, Germany), Andreas J. Forstner (Institute of Human Genetics, School of Medicine \& University Hospital Bonn, University of Bonn, Bonn, Germany), Clyde Francks (Language and Genetics Department, Max Planck Institute for Psycholinguistics, Nijmegen, The Netherlands), Barbara Franke (Department of Human Genetics, Radboud university medical center, Nijmegen, The Netherlands), David C. Glahn (Department of Psychiatry, Yale University School of Medicine, New Haven, USA), Randy L. Gollub (Department of Psychiatry, Massachusetts General Hospital, Boston, USA), Hans J. Grabe (German Center for Neurodegenerative Diseases Rostock/Greifswald, Greifswald, Germany), Oliver Gruber (Section for Experimental Psychopathology and Neuroimaging, Department of General Psychiatry, Heidelberg University Hospital, Heidelberg, Germany), Asta K. Håberg (Department of 
Neuroscience, Norwegian University of Science and Technology, Trondheim, Norway), Ahmad R. Hariri (Department of Psychology and Neuroscience, Duke University, Durham, USA), Catharina A. Hartman (Department of Psychiatry, University Medical Center Groningen, University of Groningen, Groningen, The Netherlands), Ryota Hashimoto (Molecular Research Center for Children's Mental Development, United Graduate School of Child Development, Osaka University, Suita, Japan), Andreas Heinz (Division of Mind and Brain Research, Department of Psychiatry and Psychotherapy, Campus Charité Mitte, Charité - Universitätsmedizin Berlin, Berlin, Germany), Manon H.J. Hillegers (Department of Child and Adolescent Psychiatry/Psychology, Erasmus Medical Center-Sophia Children's Hospital, Rotterdam, The Netherlands), Pieter J. Hoekstra (Department of Psychiatry, University Medical Center Groningen, University of Groningen, Groningen, The Netherlands), Avram J. Holmes (Department of Psychology, Yale University, New Haven, USA), L. Elliot Hong (Department of Psychiatry, Maryland Psychiatry Research Center, University of Maryland School of Medicine, Baltimore, USA), William D. Hopkins (Neuroscience Institute, Georgia State University, Atlanta, USA), Hilleke E. Hulshoff Pol (Department of Psychiatry, Brain Center Rudolf Magnus, University Medical Center Utrecht, Utrecht University, Utrecht, The Netherlands), Terry L. Jernigan (Center for Human Development, University of California San Diego, La Jolla, USA), Erik G. Jönsson (Centre for Psychiatric Research, Department of Clinical Neuroscience, Karolinska Institutet, Stockholm, Sweden), René S. Kahn (Department of Psychiatry, Icahn School of Medicine at Mount Sinai, New York, USA), Martin A. Kennedy (Department of Pathology and Biomedical Science, University of Otago, Christchurch, Christchurch, New Zealand), Tilo T.J. Kircher (Department of Psychiatry and Psychotherapy, Philipps-University Marburg, Marburg, Germany), Peter Kochunov (Department of Psychiatry, Maryland Psychiatry Research Center, University of Maryland School of Medicine, Baltimore, USA), John B.J. Kwok (Neurogenetics and Epigenetics, Brain and Mind Centre, The University of Sydney, Sydney, Australia), Stephanie Le Hellard (NORMENT - K.G. Jebsen Centre for Psychosis Research, Department of Clinical Science, NORMENT University of Bergen, Bergen, Norway), Nicholas G. Martin (Genetic Epidemiology, QIMR Berghofer Medical Research Institute, Brisbane, Australia), Jean - Luc Martinot (INSERM Unit 1000 - Neuroimaging \& Psychiatry, Paris Sacaly University, Gif sur Yvette, France), Colm McDonald (Centre for Neuroimaging \& Cognitive Genomics, National University of Ireland Galway, Galway, Ireland), Katie L. McMahon (Herston Imaging Research Facility, School of Clinical Sciences, Queensland University of Technology, Brisbane, Australia), Andreas MeyerLindenberg (Department of Psychiatry and Psychotherapy, Central Institute of Mental Health, Medical Faculty Mannheim, Heidelberg University, Mannheim, Germany), Rajendra A. Morey (Duke UNC Brain Imaging and Analysis Center, Duke University Medical Center, Durham, USA), Lars Nyberg (Department of Integrative Medical Biology, Umeå University, Umeå, Sweden), Jaap Oosterlaan (Emma Children's Hospital, Academic Medical Center, Amsterdam, The Netherlands), Roel A. Ophoff (Center for Neurobehavioral Genetics, University of California Los Angeles, Los Angeles, USA), Tomas Paus (Holland Bloorview Kids Rehabilitation Hospital, Bloorview Research Institute, University of Toronto, Toronto, ON, Canada), Zdenka Pausova (The Hospital for Sick Children, University of Toronto, Toronto, Canada), Brenda W.J.H. Penninx (Psychiatry, Amsterdam UMC Vrije Universiteit, Psychiatry, The Netherlands), Tinca J.C. Polderman (Complex Trait Genetics, Center for Neurogenomics and Cognitive Research, Vrije Universiteit Amsterdam, Amsterdam, The Netherlands), Danielle Posthuma (Department of Biological Psychology, Vrije Universiteit Amsterdam, Amsterdam, The Netherlands), Marcella Rietschel (Department of Genetic Epidemiology in Psychiatry, Central Institute of Mental Health, Medical Faculty Mannheim, Heidelberg University, 
Mannheim, Germany), Joshua L. Roffman (Department of Psychiatry, Massachusetts General Hospital, Boston, USA), Laura M. Rowland (Department of Psychiatry, Maryland Psychiatry Research Center, University of Maryland School of Medicine, Baltimore, USA), Perminder S. Sachdev (Centre for Healthy Brain Ageing, University of New South Wales, Sydney, Australia), Philipp G. Sämann (, Max Planck Institute of Psychiatry, Munich, Germany), Gunter Schumann (Social, Genetic and Developmental Psychiatry Centre, King's College London, London, UK), Kang Sim (General Psychiatry, Institute of Mental Health, Singapore, Singapore), Sanjay M. Sisodiya (Department of Clinical and Experimental Epilepsy, University College London, London, UK), Jordan W. Smoller (Psychiatric and Neurodevelopmental Genetics Unit, Center for Genomic Medicine, Massachusetts General Hospital, Boston, USA), Iris E. Sommer (Department of Medical and Biological Psychology, University of Bergen, Bergen, Norway), Beate St Pourcain (MRC Integrative Epidemiology Unit, Department of Population Health Sciences, Bristol Medical School, Bristol, UK), Dan J. Stein (Department of Psychiatry and Mental Health, University of Cape Town, Cape Town, South Africa), Arthur W. Toga (Laboratory of Neuro Imaging, Mark and Mary Stevens Neuroimaging and Informatics Institute, Keck School of Medicine, University of Southern California, Los Angeles, USA), Julian N. Trollor (Department of Developmental Disability Neuropsychiatry, University of New South Wales, Sydney, Australia), Nic J.A. Van der Wee (Department of Psychiatry, Leiden University Medical Center, Leiden, The Netherlands), Dennis van't Ent (Department of Biological Psychology, Vrije Universiteit Amsterdam, Amsterdam, The Netherlands), Henry Völzke (Institute for Community Medicine, University Medicine Greifswald, Greifswald, Germany), Henrik Walter (Division of Mind and Brain Research, Department of Psychiatry and Psychotherapy, Campus Charité Mitte, Charité Universitätsmedizin Berlin, Berlin, Germany), Bernd Weber (Institute of Experimental Epileptology and Cognition Research, University Hospital Bonn, Bonn, Germany), Daniel R. Weinberger (Lieber Institute for Brain Development, Baltimore, USA), Margaret J. Wright (Queensland Brain Institute, University of Queensland, St Lucia, Australia), Juan Zhou (Center for Cognitive Neuroscience, Neuroscience and behavioral disorders program, Duke-National University of Singapore Medical School, Singapore, Singapore), Jason L. Stein (Department of Genetics \& UNC Neuroscience Center, University of North Carolina at Chapel HIII, Chapel Hill, USA), Paul M. Thompson (Imaging Genetics Center, Mark and Mary Stevens Neuroimaging and Informatics Institute, Keck School of Medicine of USC, University of Southern California, Los Angeles, USA), Sarah E. Medland (Psychiatric Genetics, QIMR Berghofer Medical Research Institute, Brisbane, Australia). 